\title{
Evolution of an urban transportation system in emergency conditions: analysis through a pseudo-dynamic assignment model
}

\author{
A. Vitetta \& P. Velonà \\ Department of Computer Science, Mathematics, Electronics and \\ Transportation, Mediterranea University of Reggio Calabria, Italy.
}

\begin{abstract}
In this paper a transport system in a case of evacuation is studied by using the traditional methodologies of the static analysis of the transport systems, by verifying its compatibility and effectiveness under conditions of performance to the limit of saturation and by looking for an approach including an analysis which is not so different from the static one. This methodology, called pseudo-dynamic methodology, allows the overcoming of those limits the static analysis sets when it is applied to the study of a phenomenon having a natural dynamic.
\end{abstract}

\section{Introduction}

The problem to verify the performance of a transport system, in case the general mobilization becomes necessary in a city or an area including various urban conglomerations, is of remarkable interest for the definition of mathematical instruments supporting decisions, for an optimal planning of interventions on both a strategic and an operational level. The concept of general mobilization of a city is expressed and synthesized, in the technical language of the field, using the term "evacuation".

The importance of a quantitative analysis results from the possibility to support, through numerical data, hypotheses that, otherwise, would not be verifiable without huge social and economic costs.

The approaches commonly used for the analysis of the performance of a transport system are two: the simulation approach and the optimization approach. The former, defined as "what if" approach, allows to verify the interaction between 


\section{Environmental Health Risk}

the various components of the transport system and to establish their compatibility, once the basic hypotheses of and the setting to be simulated are defined.

The latter, defined as "what to" approach [1], allows determining, as an output of the problem, one or more components of the transport system in order to assure the achievement of fixed objectives.

The typical approach of the "what if" models is the equilibrium static one, in which deterministic equilibrium assignment, DUE, ([2], [3], [4], [5], [6]) can be distinguished from stochastic equilibrium assignment, SUE, ([7], [8]).

The "what if" static assignment models are formulated in the hypothesis of absence of within-day dynamics and, therefore, of absence of over-saturation phenomena which cannot be analyzed in a static context. This means that the static approach is inadequate when it is necessary to know the performance of a transport system in case of evacuation.

If the usual equilibrium approach cannot be used, it will be necessary to follow either a dynamic ([9], [10], [11]) or a pseudo-dynamic approach ([12], [13], [14]).

Although it makes use of the typical instruments of the static analysis, the pseudo-dynamic approach allows a better perception of the problem that is being studied. It can be used within the methods of demand planning for the analysis of a city in evacuation. In this paper the study of a transport system in case of evacuation is proposed by using the traditional methodologies of the static analysis of the transport systems, by verifying its compatibility and effectiveness in conditions of performance to the limit of saturation and by looking for an approach to the problem that allows the use of an analysis not very different from that static one. This methodology, called "pseudo-dynamic", allows the overcoming of those limits the static analysis imposes when it is applied to the study of a phenomenon being characterized by a dynamic evolution.

\section{Models}

Both the "what if" and the "what to" can be divided into four fundamental steps of analysis:

- analysis of the territory;

- analysis of the supply;

- analysis of the demand;

- analysis of the supply-demand interaction.

It is important to specify that, while the "what if" models allow to verify the effects of the variations undergone by the elements of a transport system, the "what to" models allow to solve design problems and therefore to determine the configuration of the elements of the system satisfying very precise conditions.

Within the hypotheses being the basis of the application of both the "what if" and "what to" models to the case of the evacuation of a city, it is necessary to add the distinction between user strategy and transport system manager strategy. The 
user tries to look after his/her own interests for the protection of his/her safety and the one of his/her most intimate relatives and not for the defence of the common good; on the contrary, the transport system manager tries to achieve a series of objectives and aims for the protection of the common interest. In some cases, the common interest can contrast with the objectives of a particular group of users. A third figure can be added to the user and the manager: the analyst, having the task to simulate the settings proposed by the manager through the use of instruments he masters.

Two cases can occur:

- the manager proposes a setting and the analyst verifies it through "what if" models and/or procedures, taking into account or not the user behavioural hypotheses;

- the manager asks to obtain as output variables elements of the demand and/or of the supply; in such a case the analyst makes use of "what to" models and/or procedures however including "what if" models. Even in this case the analyst can take into account or not the user behavioural hypotheses. Later in this paper the aspect concerning transport demand planning and organization through "what to" models (demand management) will be developed postponing the analysis of "what if" models and of supply design models (network design) to other contexts.

\section{1 "What to" models}

The design of a transport system (network design and/or demand management) consists in determining the optimal configuration of one or more subsystems making it (supply and/or demand respectively), considering the optimization of various objectives. In this paper the case of an emergency evacuation (carried out in a limited time) and of a prearranged evacuation (carried out in a sufficiently long time) of a transport network were considered, taking into account the two following objectives:

- for the emergency evacuation (EE), the aim is to maximize the number of users to evacuate in a relatively short time (an hour);

- for prearranged evacuation (EP), the aim is to minimize the time of total evacuation supposing to dispose of a longer time.

From a mathematical point of view, in the general case the design model can be formulated as an optimal model in which there are:

- design variables: $\mathbf{d}_{\mathbf{D}}$ (distribution vector), $\mathbf{d}_{\mathrm{TIME}(\mathrm{h}), \mathbf{b}}$ (demand vector between the various od pairs in the time band $h$ ), $\mathbf{D}_{\text {TIME,D }}$ (matrix having $\mathbf{d}_{\mathbf{T I M E}(\mathrm{h}), \mathrm{D}}$ as column vector), $\mathbf{d}_{\text {TIME(h) }}$ (vector of the demand expressed by the various origins or in the time band $h$ ), $\mathbf{D}_{\text {TIME }}$ (matrix having $\mathbf{d}_{\text {TIME(h) }}$ as column vector);

- objective function: total time of evacuation or spent by the users on the network (to be minimized), number of users to evacuate from the risk area 


\section{Environmental Health Risk}

(to be maximized) in a fixed interval of time, total time of run (to be minimized);

- design constraints: user behaviour, constraints of capacity, constraints of budget, time of evacuation, etc.

In its general expression the model has not a simple solution and can be solved on several levels (optimal multi-level) by means of heuristic algorithms. In this paper the demand distribution in the assembly centres and in various time bands will be considered as simplified cases of the design meant as demand management.

\subsubsection{Demand management model for the demand distribution in several assembly centres}

The problem is to determine the percentages of distribution of the users in the various assembly centres.

The model is introduced as follows:

Design variables:

$$
\begin{aligned}
& \mathbf{d}_{\text {TIME(h),D }} \\
& \mathbf{D}_{\text {TIME,D }}
\end{aligned}
$$

Objective function:

max users evacuated in a fixed time $h(E E)$

min total time of evacuation (EP):

constraints: of user behaviour (hypothesis on the adopted assignment model), of capacity.

\subsubsection{Demand management model for departure time}

The developed model allows determining the lowest number of time bands necessary in order that all the users coming from an origin zone can reach a certain destination, on condition that the flow distribution, caused by the demand assigned in each band, is compatible with the capacity values. In such a sense, a time distribution of the demand, expressed by each origin or distributed per od pair, is carried out.

If a purely static approach were used, a given initial demand vector should be assigned at the same time in one reference period. Flows greater than capacity would be obtained and there would not be an exhaustive vision of the state of the system because, in that case, it would be as if static assignment blocked the whole dynamic evolution of the system in one moment.

It is obvious that the problem of queue formation and of over-saturation arise and that they cannot be analysed through a static approach. It is necessary to work under or even to the limit of capacity in order to obtain results being acceptable and, however, representative of the state of the system in a fixed period (for example an hour).

If, though maintaining equilibrium approach logic, the use of a dynamic approach is required, various time bands (procedures take into account hours) 
can be created and the demand vector, to be assigned to each of them, can be designed in order to reach network saturation. In mathematical terms the problem could be formulated through a "what to" model as follows:

Design variables:

$$
\begin{aligned}
& \mathbf{d}_{\text {TIME(h), }}, \mathbf{d}_{\text {TIME(h),D }} \\
& \mathbf{D}_{\text {TIME }}, \mathbf{D}_{\text {TIME,D }}
\end{aligned}
$$

objective function:

max users evacuated in a fixed time $h$

min time of evacuation

constraints: of user behaviour (hypothesis on the adopted assignment model), of capacity.

\section{Procedures}

Some procedures were considered in order to realize the management of the distribution and of the departure time. The objective of the studied procedures consists in the search of an acceptably realistic solution which could be adopted in case of emergency and prearranged evacuation.

In the case of the management of the distribution the aim is to determine the percentages of distribution of the demand in the various assembly centres in a fixed interval (EE) (for example an hour) or in each time interval the whole process of evacuation (EP) is divided into.

In the case of the management of the departure time either the highest number of departures from an emission zone, or transfers between an od pair with fixed or non fixed distribution and in a fixed time interval (EE), or a subdivision of departures in fixed time bands (EP) with a possible search of the optimal distribution in each band, can be determined.

It can be deduced that a synergy exists between the management of the distribution and of the departure time. The proposed procedures realize such a synergy realizing, at the same time, the two levels of planning. In particular, in the case of the prearranged evacuation, they work through a pseudo-dynamic assignment because they take into account the dynamic evolution of the system searching for a time distribution of the demand in several bands, but, at the same time, using an internal procedure of static assignment for flow determination.

The proposed procedures consider the topological network structure as their input and give as their output:

- the demand (d $\left.\mathbf{d}_{\text {TIME(1),D }}\right)$ which must be distributed in the assembly centres considering 1-hour interval, (EE);

- the total demand which must be emitted ( $\mathbf{D}_{\text {TIME }}$ ) or which must be distributed ( $\left.\mathbf{D}_{\mathbf{T I M E}, \mathbf{D}}\right)$ in the assembly centres in the various time bands $\mathrm{h}$, (EP).

The procedures used later in this paper include the following subprocedures, for some of which input and output demand vectors were respectively indicated as dinput and doutput: 


\section{Environmental Health Risk}

- FICTITIOUS_NODE, consists in connecting the assembly centres to one node called "fictitious destination"; in such a way it can be assumed that the user destination is one and shifted to the fictitious node;

- STATIC_LOADING( $\left.\mathbf{d}_{\text {input }}, \mathbf{d}_{\text {output }}\right)$, is a heuristic procedure allowing to determine the demand vector to be assigned to a time band $\mathrm{h}$, consistently with the supply characteristics; it is characterized by the following steps: step 0 assignment of an initial demand vector, $\left(\mathbf{d}_{\text {input }}\right)$;

step1 for each od pair, calculation of the path used the users and of the link with the highest saturation level;

step 2 increment of the demand value for the pairs with the highest saturation level being lower than a fixed threshold $(1 \div 1.1)$; decrement of the demand value for the pairs with the highest saturation level being higher than the above mentioned threshold;

step 3 assignment of the new demand vector;

step 4 if the highest saturation level on the whole network exceeds a fixed value $(1,1)$ or the highest fixed value of iterations is reached $(300 \div 600)$, the procedure will finish and the iteration to which the demand vector ( $\mathbf{d}_{\text {output }}$ ) with the highest total demand assigned to all pairs corresponds, will be searched for; otherwise procedure strarts again from step 2 .

- ASSIGNMENT( $\left(\mathbf{d}_{\text {input }}\right)$, implements an assignment model, DUE.

- ASS_FICT_NODE $\left(\mathbf{d}_{\text {input }}, \mathbf{d}_{\text {output }}\right)$, allows to obtain, after assignment, the vector of the demand distributed in the assembly centres; it is characterized by the following steps:

step 0 it applies the FICTITIOUS NODE procedure; if that has been already applied, it will go directly to step 1;

step 1 it assigns an emitted demand vector $\left(\mathbf{d}_{\text {input }}\right)$;

step 2 it calculates the percentages of demand in the various assembly centres for each origin, defining, after the assignment, the vector of the demand distributed $\left(\mathbf{d}_{\text {output }}\right)$ in the assembly centres.

Procedures were considered in order to simulate, through their appropriate combination, emergency and prearranged evacuation.

In the former case (EE), the greatest number of users to evacuate in 1-hour interval with fixed distribution (Procedure 1) and the greatest number of users to evacuate in 1-hour interval together with their distribution (Procedure 2) were searched for. It should be noticed that, in Procedure 1, the distributed demand vector is obtained through a Logit model depending on the parameter $\beta, \mathbf{d}_{\mathbf{D}}(\beta)$.

In the latter case (EP), total demand was assigned by distributing it on several time bands (pseudo-dynamic assignment) using the three following procedures.

In Procedure 3, in each time band network is loaded to the limit of saturation, so determining the corresponding demand volume to emit. STATIC LOADING(·) procedure is applied repeatedly, until all the demand is emitted. Total evacuation time will be given by the number of bands being necessary to assign to all the 
demand. Moreover, for each time band $\mathrm{h}$, distribution after assignment can be obtained by applying the ASS_FICT_NODE(d $\mathbf{d}_{\text {TIME(h) }} \mathbf{d}_{\text {TIME(h), } \mathbf{p})}$ procedure (management of the distribution). In Procedure 4, FICTITIOUS_NODE and ASSIGNMENT() procedures are applied first in order to estimate the highest saturation level on the network; then the emitted demand is divided by the integer corresponding to such a saturation level and the compatibility of the new demand with the supply is verified. The total evacuation time will be given by the number of bands being necessary to assign all the demand. As in the case of Procedure 3, for each time band, the distribution after assignment can be obtained by applying the ASS_FICT_NODE $\left(\mathbf{d}_{\text {TIME(h) }}, \mathbf{d}_{\text {TIME(h), }}\right)$ procedure (management of the distribution).

In Procedure 5, ASS_FICT_NODE() procedure is applied first, in order to estimate the highest saturation level on the network; then the distributed demand is divided by the integer corresponding to such a saturation level, and the compatibility of the new demand with the supply is verified. The total evacuation time will be given by the number of bands being necessary to assign all the demand. Moreover, in comparison with the previous case, a management of the distribution is carried out first within the procedure and then time distribution is obtained.

\section{Applications to the town of Reggio Calabria}

The application allowed to compare the results obtained through the five identified procedures (Procedure 1, Procedure 2, Procedure 3, Procedure 4, Procedure 5) in terms of number of evacuated people after an hour (Procedure 1, Procedure 2, Procedure 3, Procedure 4, Procedure 5) and of total time of evacuation (Procedure 3, Procedure 4, Procedure 5), considering a DUE assignment model.

Procedures were applied to the town of Reggio Calabria, from which the graph of the road network reserved to aid services was previously extracted.

The transport network is made up of 382 nodes, 883 links; the territory is divided into 30 traffic zones, each of them being represented by a source internal centroid, that is by a point where the emissions of the zone are concentrated. The assembly centres are 8 and the total demand to be evacuated is of 102333 vehicles.

The results obtained through the application of the first two procedures (Procedure 1 e Procedure 2), carried out in order to simulate the evacuation in emergency conditions, show that (table 1):

- It is not suitable to send users to the nearest gathering centre $(\beta=100)$ because the number of total evacuated people is 2.5 times lower than the highest value obtained through Procedure $1(\beta=2)$;

- If destination is not fixed beforehand, but, during simulation, the user is given the opportunity to dispose of a larger set of alternatives (that is obtained by connecting the assembly centres to a fictitious node and, therefore, considering just one destination for each origin), a better 


\section{Environmental Health Risk}

distribution on the network and a greater number of evacuated people after an hour can be obtained (Procedure 2);

The results of the three other procedures (Procedure 3, Procedure 4, Procedure 5 ), carried out in order to simulate the prearranged evacuation, show that (table 2):

- Having an initial high value of evacuated users causes some problems later on, when the od pairs using high capacity paths and links have exhausted emissions and only the most critical pairs have remained tending to reduce the increases in demand to be assigned in the following iterations (Procedure $3)$;

- The uniform distribution in the different time bands causes an increase in the number of evacuated users (Procedure 4 and Procedure 5);

- If the distribution in the assembly centres is fixed before the one in the different time bands (Procedure 5), total time evacuation will be reduced in comparison with the case where the two (space and time) distributions are carried out at the same time (Procedure 4), although time distribution is uniform in both cases.

Table 1: Results of Procedure 1 and Procedure 2 with DUE (EE)

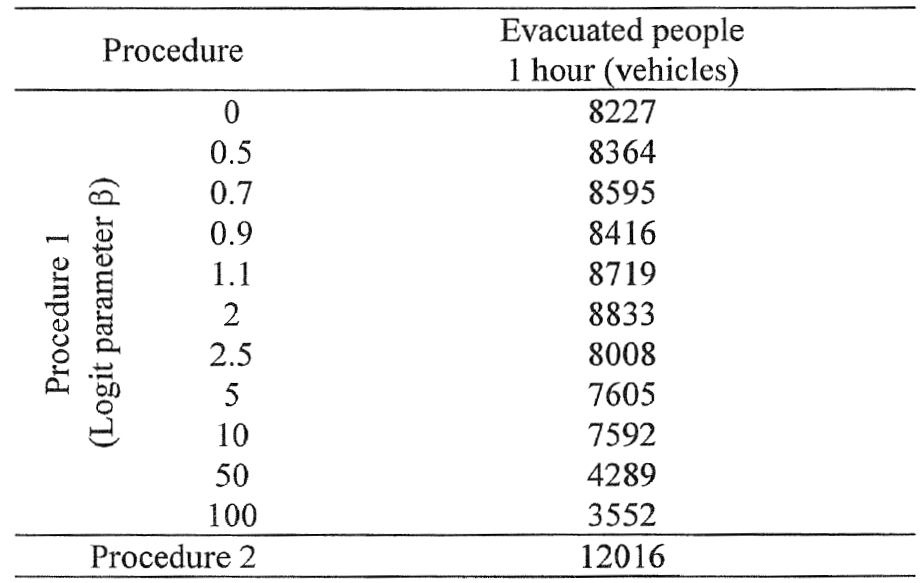

Passing from a non uniform time distribution, where paths are not imposed (Procedure 3), to a uniform distribution with a double-level design of space distribution first and then of time distribution (Procedure 5), the total time of evacuation is reduced of 6 hours.

An application to a small town (Zafferana Etnea, Italy) is described in [15], where the road trasportation system is simulated for different configurations of supply (different number and location of assembly centers) and demand (different distributions among the assembly centers), according to a "what if" approach. 
Table 2: Results Procedure 3, Procedure 4 and Procedure 5 with DUE (EP)

\begin{tabular}{ccccc}
\hline Procedure & $\begin{array}{c}\text { Evacuated } \\
\text { people } \\
1 \text { hour } \\
\text { (vehicles) }\end{array}$ & $\begin{array}{c}\text { Average } \\
\text { (vehicles) }\end{array}$ & $\begin{array}{c}\text { Variance } \\
\left(\text { vehicles }^{2}\right)\end{array}$ & $\begin{array}{c}\text { Total } \\
\text { time } \\
\text { (hours) }\end{array}$ \\
\hline Procedure 3 & 12016 & 4449 & 9541087 & 23 \\
\hline Procedure 4 & 4651 & 4651 & 0.0 & 22 \\
\hline Procedure 5 & 6019 & 6019 & 0.0 & 17 \\
\hline
\end{tabular}

\section{Conclusions}

The analysis proposed in this paper uses the typical instruments of the static approach and defines procedures which can combine a moment of verification, the one allowing determining the time of network evacuation, with a design moment, the one when it is necessary to determine demand distribution in the various assembly centres and in several time bands.

Applications confirm the possibility of a pseudo-dynamic approach in order to obtain an estimation of the time of evacuation, though reaffirming the impossibility to analyze flow conditions during the phenomenon.

The results obtained on the town of Reggio Calabria reveal the possibility to determine an optimal space distribution so that the number of evacuated users in an hour can be maximized; to determine a space and time distribution (pseudodynamic assignment) so that the total time of evacuation can be reduced. It has been noticed that "designing" the distribution in the assembly centres and in several time bands in a uniform way causes a reduction of 6 hours (about 30\%) in the total time of evacuation in comparison with the case where just a non uniform time distribution is designed.

Future developments are tied to the possibility to define exact procedures including the proposed demand management model for the departure time and are also tied to the use of a purely dynamic approach for a relation of interaction and exchange of information between static and dynamics aiming at a better comprehension of the problems to study.

\section{References}

[1] Cascetta, E., Transportation systems engineering: theory and methods, Kluwer, 2001.

[2] Beckman, M., McGuire, C.B. \& Winsten, C.B., Studies in the economics of transportation. Yale University Press, New Haven, CT, 1956.

[3] Dafermos, S., An extended traffic assignment model with applications to twoway traffic. Transp. Sci., vol.5, pp. 366-389, 1971.

[4] Dafermos, S., Traffic equilibrium and Variational Inequalities. Transp. Sci., vol.14 (1), pp. 42-54, 1980. 
226 Environmental Health Risk

[5] Dafermos, S., The general multimodal network equilibrium problem with elastic demand. Networks, No 12, pp. 57-72, 1982.

[6] Smith, M.J., Existence, Uniqueness and Stability of Traffic Equilibrium. Transpn Res. 13B, 1979

[7] Daganzo, C.F. \& Sheffi, Y., On Stochastic Models of Traffic Assignment. Trans. Sci. 11(3), 253-274, 1977.

[8] Sheffi, Y. \& Powell, W.B., A comparison of stochastic and deterministic traffic assignment over congested network. Transportation Research, Vol.15B, 53-64, 1981

[9] Di Gangi, M., Luongo, A. \& Polidoro, R., Una procedura di carico dinamico per la valutazione dei piani di evacuazione. In Metodi e Tecnologie dell'Ingegneria dei Trasporti - Seminario 1999 a cura di G.E. Cantarella e F. Russo, FrancoAngeli, 2001.

[10]Di Gangi, M. \& Velonà, P., Use of a mesoscopic dynamic assignment model for approaching the evolution of an urban transportation system in emergency conditions. In this book, 2003.

[11]Musolino, G. \& Vitetta, A., Microscopic approach for the evaluation of a urban transport system in emergency condition. In this book, 2003.

[12]Russo, F. \& Vitetta, A., Urban road transportation analysis in emergency conditions: models and algorithms. Proceedings of Urban Transport 2000, Wessex Institute of Technology. Sucharov e Brebbia ed., WIT Press Boston, pp. 533-542, 2000.

[13]Russo, F. \& Vitetta, A., Urban transportation system analysis in emergency conditions. In this book, 2003.

[14]Velonà, P. \& Vitetta, A., Procedure di assegnazione pseudo dinamica per la valutazione del tempo di evacuazione di una rete di trasporto. Atti INPUT 2001, Isole Tremiti, 2001.

[15]Di Gangi, M., Musolino, G., Russo, F., Velonà, P. \& Vitetta, A. Analysis and comparison of several urban road transportation assignment models. In this book, 2003. 